JOURNAL OF

SYMPLECTIC GEOMETRY

Volume 12, Number 2, 215-236, 2014

\title{
SYMPLECTIC MANIFOLDS AND COHOMOLOGICAL DECOMPOSITION
}

\author{
Daniele Angella And Adriano Tomassini
}

Given a closed symplectic manifold, we study when the Lefschetz decomposition induced by the $\mathfrak{s l}(2 ; \mathbb{R})$-representation yields a decomposition of the de Rham cohomology. In particular, this holds always true for the second de Rham cohomology group, or if the symplectic manifold satisfies the Hard Lefschetz Condition.

\section{Introduction}

Compact Kähler manifolds have special cohomological properties: from the complex point of view, the Hodge decomposition theorem states that the complex de Rham cohomology groups decompose as direct sum of the Dolbeault cohomology groups, and from the symplectic side the Hard Lefschetz theorem provides a decomposition of the de Rham cohomology as direct sum of primitive cohomology groups. Such decompositions do not hold anymore for general non-Kähler complex manifolds.

To the purpose of generalizing the above cohomological complex-type decomposition on an arbitrary almost-complex manifold $(X, J)$, T.-J. Li and $\mathrm{W}$. Zhang have introduced in $[\mathbf{2 1}]$ the subgroups $H_{J}^{(p, q),(q, p)}(X ; \mathbb{R}) \subseteq$ $H_{d R}^{\bullet}(X ; \mathbb{R})$ (respectively, $H_{J}^{(p, q)}(X) \subseteq H_{d R}^{\bullet}(X ; \mathbb{C})$ ), formed by the real (respectively, complex) de Rham cohomology classes having representatives of pure type $(p, q)$ (we refer also to $[\mathbf{2}, \mathbf{3}, \mathbf{1 7}]$ for further results concerning these subgroups). In [15], T. Drăghici, T.-J. Li, and W. Zhang have proved that any closed four-dimensional manifold endowed with an almostcomplex structure $J$ satisfies the decomposition $H_{d R}^{2}(X ; \mathbb{R})=H_{J}^{(1,1)}(X ; \mathbb{R}) \oplus$ $H_{J}^{(2,0),(0,2)}(X ; \mathbb{R})$, which can be regarded as a Hodge decomposition for nonKähler 4-manifolds. This decomposition does not hold true in higher dimension; see [17, Example 3.3].

In [8], Brylinski proposed a Hodge theory for closed symplectic manifolds $(X, \omega)$ : in this context, Mathieu in $[\mathbf{2 4}]$, and Yan in $[\mathbf{3 2}]$, proved that any 
de Rham cohomology class admits a symplectically harmonic representative (i.e., a form being both $\mathrm{d}$-closed and $\mathrm{d}^{\Lambda}$-closed, where $\mathrm{d}^{\Lambda}\left\lfloor_{\wedge^{k} X}:=(-1)^{k+1} \star_{\omega}\right.$ $\mathrm{d} \star_{\omega}$, and $\star_{\omega}$ is the symplectic- $\star$-operator) if and only if the Hard Lefschetz Condition is satisfied.

Recently, Tseng and Yau, in [29, 30] (see also [31]), have introduced new cohomologies for symplectic manifolds $(X, \omega)$ : among them, in particular, they have defined and studied

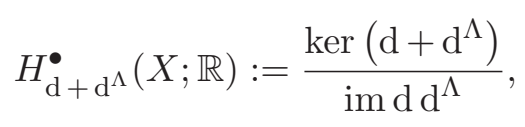

developing a Hodge theory for such cohomology; $H_{\mathrm{d}+\mathrm{d}^{\Lambda}}^{\bullet}(X ; \mathbb{R})$ can be interpreted as the symplectic counterpart to the Bott-Chern cohomology of a complex manifold; see [31]. (As regards the Bott-Chern cohomology and its relation with the cohomological properties of a compact complex manifold, we refer to [4], where the problem whether the Bott-Chern cohomology groups induce a decomposition of the de Rham cohomology is studied, and a characterization of compact complex manifolds satisfying the $\partial \bar{\partial}$-Lemma is given.) Furthermore, they have studied the dual currents of Lagrangian and co-isotropic submanifolds, and they have defined a homology theory on co-isotropic chains, which turns out to be naturally dual to a primitive cohomology.

Inspired also by their work, Lin has developed in [22] a new approach to the symplectic Hodge theory.

In the present paper, we focus on cohomological properties of closed symplectic manifolds $(X, \omega)$. Denote by

$$
\begin{aligned}
H_{\omega}^{(r, s)}(X ; \mathbb{R}):= & \left\{\left[L^{r} \beta^{(s)}\right] \in H_{d R}^{2 r+s}(X ; \mathbb{R}): \beta^{(s)} \text { is a primitive } s \text {-form }\right\} \\
& \subseteq H_{d R}^{\bullet}(X ; \mathbb{R})
\end{aligned}
$$

and by

$$
\begin{aligned}
H_{(r, s)}^{\omega}(X ; \mathbb{R}) & :=\left\{\left[L^{r} \beta_{(s)}\right] \in H_{-2 r+s}^{d R}(X ; \mathbb{R}): \beta_{(s)} \text { is a primitive } s \text {-current }\right\} \\
& \subseteq H_{\bullet}^{d R}(X ; \mathbb{R})
\end{aligned}
$$

where $L: \wedge^{\bullet} X \rightarrow \wedge^{\bullet+2} X$ is defined by $L \alpha:=\omega \wedge \alpha$, and $L: \mathcal{D}_{\bullet} X \rightarrow \mathcal{D}_{\bullet-2} X$ is induced by duality, where $\mathcal{D} \cdot X$ denotes the complex of currents on $X$.

We are concerned in studying when the above subgroups yield a direct sum decomposition of the de Rham cohomology, respectively, of the de Rham homology. In this matter, we prove the following result, which can be regarded as the symplectic counterpart to $[\mathbf{1 5}$, Theorem 2.3] by T. Drăghici, Li, and Zhang in the almost-complex case. 
Theorem 2.6. Let $X$ be a closed manifold endowed with a symplectic structure $\omega$. Then

$$
H_{d R}^{2}(X ; \mathbb{R})=H_{\omega}^{(1,0)}(X ; \mathbb{R}) \oplus H_{\omega}^{(0,2)}(X ; \mathbb{R}) .
$$

In particular, if $\operatorname{dim} X=4$, then

$$
H_{d R}^{\bullet}(X ; \mathbb{R})=\bigoplus_{r \in \mathbb{N}} H_{\omega}^{(r, \bullet-2 r)}(X ; \mathbb{R})
$$

and

$$
H_{\bullet}^{d R}(X ; \mathbb{R})=\bigoplus_{r \in \mathbb{N}} H_{(r, \bullet+2 r)}^{\omega}(X ; \mathbb{R})
$$

If $(X, \omega)$ satisfies the Hard Lefschetz Condition, then $H_{d R}^{\bullet}(X ; \mathbb{R})$, respectively $H_{\bullet}^{d R}(X ; \mathbb{R})$, decomposes as direct sum of the subgroups $H_{\omega}^{(\bullet \bullet \bullet)}(X ; \mathbb{R})$, respectively, $H_{(\bullet . \bullet}^{\omega}(X ; \mathbb{R})$; see Corollary 2.5 .

Then we specialize on solvmanifolds, namely, compact quotients of solvable Lie groups, showing a Nomizu theorem for solvmanifolds of completely solvable type; see Proposition 3.3.

The paper is organized as follows. In Section 1, we recall the basic facts concerning Lefschetz decomposition (both for differential forms, for currents, and for cohomologies) and Hodge theory on closed symplectic manifolds, in particular with the aim to fix the notations. In Section 2, we introduce and study the subgroups $H_{\omega}^{(\bullet \bullet \bullet)}(X ; \mathbb{R})$, proving the symplectic cohomological decomposition in Theorem 2.6. In Section 3, we study symplectic cohomology decomposition for solvmanifolds, providing several explicit examples and computing the symplectic cohomology groups in such cases.

\section{Preliminaries on Hodge theory for symplectic manifolds}

We recall here some notions and results concerning Hodge theory for symplectic manifolds, referring to $[8,9,22,24,29,30,32]$.

1.1. Primitive forms and Lefschetz decomposition. Let $(V, \omega)$ be a $2 n$-dimensional symplectic vector space and denote by $\left\{e_{1}, \ldots, e_{2 n}\right\}$ a Darboux basis of $V$ for $\omega$, i.e., $\omega=\sum_{i=1}^{n} e^{i} \wedge e^{n+i}$, where $\left\{e^{1}, \ldots, e^{2 n}\right\}$ is the dual basis of $\left\{e_{1}, \ldots, e_{2 n}\right\}$. Denote by $I: V \rightarrow V^{*}$ the natural isomorphism induced by $\omega$, namely $I(v)(\cdot)=\omega(v, \cdot)$, for every $v \in V$. Then $\omega$ gives rise to a bilinear form $\left(\omega^{-1}\right)^{k}$ on $\wedge^{k} V^{*}$ : on the simple elements, set

$$
\left(\omega^{-1}\right)^{k}\left(\alpha^{1} \wedge \cdots \wedge \alpha^{k}, \beta^{1} \wedge \cdots \wedge \beta^{k}\right):=\operatorname{det}\left(\omega^{-1}\left(\alpha^{i}, \beta^{j}\right)\right)_{i, j \in\{1, \ldots, k\}},
$$

where $\omega^{-1}\left(\alpha^{i}, \beta^{j}\right):=\omega\left(I^{-1}\left(\alpha^{i}\right), I^{-1}\left(\beta^{j}\right)\right)$. Note that $\left(\omega^{-1}\right)^{k}$ is skewsymmetric, respectively symmetric, according that $k$ is odd, respectively 
even. The symplectic- $\star$-operator

$$
\star_{\omega}: \wedge^{\bullet} X \rightarrow \wedge^{2 n-\bullet} X
$$

is defined requiring that, for every $\alpha, \beta \in \wedge^{k} V^{*}$,

$$
\alpha \wedge \star_{\omega} \beta=\left(\omega^{-1}\right)^{k}(\alpha, \beta) \frac{\omega^{n}}{n !},
$$

see $[\mathbf{8}$, Section 2].

Let $X$ be a $2 n$-dimensional closed manifold and let $\omega$ be a symplectic structure on $X$ (namely, a non-degenerate d-closed 2-form on $X$ ). Set $\Pi:=$ $\omega^{-1} \in \wedge^{2} T X$ the canonical Poisson bi-vector associated to $\omega$. In a Darboux chart with local coordinates $\left\{x^{1}, \ldots, x^{n}, y^{1}, \ldots, y^{n}\right\}$, if $\omega \stackrel{\text { loc }}{=} \sum_{j=1}^{n} \mathrm{~d} x^{j} \wedge \mathrm{d} y^{j}$, then $\omega^{-1} \stackrel{\text { loc }}{=} \sum_{j=1}^{n} \frac{\partial}{\partial x^{j}} \wedge \frac{\partial}{\partial y^{j}}$. Consider the $\mathfrak{s l}(2 ; \mathbb{R})$-representation on $\wedge^{\bullet} X$ given by $\langle L, \Lambda, H\rangle \subset \operatorname{End}^{\bullet}\left(\wedge^{\bullet} X\right)$, where

$$
\begin{aligned}
& L: \wedge^{\bullet} X \rightarrow \wedge^{\bullet+2} X, \alpha \mapsto \omega \wedge \alpha, \\
& \Lambda: \wedge^{\bullet} X \rightarrow \wedge^{\bullet-2} X, \quad \alpha \mapsto-\iota_{\Pi} \alpha, \\
& H: \wedge^{\bullet} X \rightarrow \wedge^{\bullet} X, \quad \alpha \mapsto \sum_{k}(n-k) \pi_{\wedge^{k} X} \alpha,
\end{aligned}
$$

(we denote the interior product with $\xi \in \wedge^{2}(T X)$ by $\iota_{\xi}: \wedge^{\bullet} X \rightarrow \wedge^{\bullet-2} X$, and, for $k \in \mathbb{N}$, the natural projection by $\left.\pi_{\wedge^{k} X}: \wedge^{\bullet} X \rightarrow \wedge^{k} X\right)$. Using the symplectic- $\star$-operator $\star_{\omega}$, one can write

$$
\Lambda=-\star_{\omega} L \star_{\omega} .
$$

The above $\mathfrak{s l}(2 ; \mathbb{R})$-representation, having finite $H$-spectrum, induces the Lefschetz decomposition on differential forms, [32, Corollary 2.6],

$$
\wedge^{\bullet} X=\bigoplus_{r \in \mathbb{N}} L^{r} \mathrm{P} \wedge^{\bullet-2 r} X,
$$

where

$$
\mathrm{P} \wedge{ }^{\bullet}:=\operatorname{ker} \Lambda
$$

is the space of primitive forms. Note (see, e.g., [20, Proposition 1.2.30(v)]) that, for every $k \in \mathbb{N}$,

$$
\mathrm{P} \wedge^{k} X=\operatorname{ker} L^{n-k+1}\left\lfloor_{\wedge^{k} X},\right.
$$

where, here and in the sequel, "[" denotes the restriction. In general, see [30, pages $7-8]$, the Lefschetz decomposition of $A^{(k)} \in \wedge^{k} X$ reads as

$$
A^{(k)}=\sum_{r \geq \max \{k-n, 0\}} \frac{1}{r !} L^{r} B^{(k-2 r)},
$$


where, for $r \geq \max \{k-n, 0\}$,

$$
B^{(k-2 r)}:=\left(\sum_{\ell \in \mathbb{N}} a_{r, \ell,(n, k)} \frac{1}{\ell !} L^{\ell} \Lambda^{r+\ell}\right) A^{(k)} \in \mathrm{P} \wedge^{k-2 r} X
$$

and, for $r \geq \max \{k-n, 0\}$ and $\ell \in \mathbb{N}$,

$$
\begin{aligned}
a_{r, \ell,(n, k)}:= & (-1)^{\ell} \cdot(n-k+2 r+1)^{2} \cdot \prod_{i=0}^{r} \frac{1}{n-k+2 r+1-i} . \\
& \times \prod_{j=0}^{\ell} \frac{1}{n-k+2 r+1+j} \in \mathbb{Q} .
\end{aligned}
$$

We recall that

$$
L\left\lfloor_{\bigoplus_{k=-1}^{n-2} \wedge^{n-k-2} X}: \bigoplus_{k=-1}^{n-2} \wedge^{n-k-2} X \rightarrow \wedge^{n-k} X\right.
$$

is injective, see [32, Corollary 2.8], and that, for every $k \in \mathbb{N}$,

$$
L^{k}: \wedge^{n-k} X \rightarrow \wedge^{n+k} X
$$

is an isomorphism, see [32, Corollary 2.7].

\subsection{Symplectic cohomologies. Set}

$$
\mathrm{d}^{\Lambda}\left\lfloor_{\wedge^{k} X}:=(-1)^{k+1} \star_{\omega} \mathrm{d} \star_{\omega}\right.
$$

for every $k \in \mathbb{N}$. Then the following basic symplectic identity holds (see, e.g., [32, Corollary 1.3]):

$$
[\mathrm{d}, \Lambda]=\mathrm{d}^{\Lambda} .
$$

As a direct consequence of $(1)$, one gets $\mathrm{d} \mathrm{d}^{\Lambda}+\mathrm{d}^{\Lambda} \mathrm{d}=0$.

Note also that, if $(J, \omega, g)$ is an almost-Kähler structure on $X$, then the symplectic- $\star$-operator $\star_{\omega}$ and the Hodge- $*$-operator $*_{g}$ are related by $\star_{\omega}=$ $J *_{g}$, and hence $\mathrm{d}^{\Lambda}=-\left(\mathrm{d}^{c}\right)^{* g}$ where $\left.\mathrm{d}^{c}:=-\mathrm{i}(\partial-\bar{\partial}).\right)$

Since $\left(d^{\Lambda}\right)^{2}=0$, one can consider, as in $[\mathbf{8}$, Section 1] and $[\mathbf{2 9}$, Section 3.1], the following cohomology:

$$
H_{\mathrm{d}^{\Lambda}}^{\bullet}(X ; \mathbb{R}):=\frac{\operatorname{ker} \mathrm{d}^{\Lambda}}{\operatorname{im\mathrm {d}^{\Lambda }},}
$$

which is isomorphic to the de Rham cohomology, since

$$
\star_{\omega}: H_{d R}^{\bullet}(X ; \mathbb{R}) \stackrel{\simeq}{\longrightarrow} H_{\mathrm{d}^{\Lambda}}^{2 n-\bullet}(X ; \mathbb{R}),
$$

by $[8$, Corollary 2.2.2]. 
In [29], looking for a symplectic counterpart to the Aeppli and BottChern cohomologies of complex manifolds (see [31] for further discussions), Tseng and Yau introduce also the $\left(\mathrm{d}+\mathrm{d}^{\Lambda}\right)$-cohomology, [29, Section 3.2],

$$
H_{\mathrm{d}+\mathrm{d}^{\Lambda}}^{\bullet}:=\frac{\operatorname{ker}\left(\mathrm{d}+\mathrm{d}^{\Lambda}\right)}{\operatorname{imdd^{\Lambda }},}
$$

and the $\left(\mathrm{d} \mathrm{d}^{\Lambda}\right)$-cohomology, $[\mathbf{2 9}$, Section 3.3],

$$
H_{\mathrm{dd}^{\Lambda}}^{\bullet}:=\frac{\operatorname{ker~d^{\Lambda }}}{\operatorname{imd}+\operatorname{imd~d}^{\Lambda}},
$$

proving in $[\mathbf{2 9}$, Corollary 3.6, Corollary 3.17$]$ that they are finite-dimensional $\mathbb{R}$-vector spaces, since $X$ is compact; more precisely, Tseng and Yau prove that, once fixed an almost-Kähler structure $(J, \omega, g)$ on $X$, these cohomologies are isomorphic to the kernel of certain $4^{\text {th }}$-order self-adjoint elliptic differential operators, see [29, Theorem 3.5, Theorem 3.16]; furthermore, the Hodge-*-operator with respect to $g$ induces the isomorphism

$$
*: H_{\mathrm{d}+\mathrm{d}^{\Lambda}}^{\bullet}(X ; \mathbb{R}) \stackrel{\simeq}{\longrightarrow} H_{\mathrm{d} \mathrm{d}^{\Lambda}}^{2 n-\bullet}(X ; \mathbb{R}),
$$

see [29, Corollary 3.25].

Moreover, it is proven in [29, Proposition 2.8] that the cohomology $H_{\mathrm{d}+\mathrm{d}^{\Lambda}}^{\bullet}(X ; \mathbb{R})$ is invariant under symplectomorphism and Hamiltonian isotopy.

The following commutation relations between the differential operators $\mathrm{d}$, $\mathrm{d}^{\Lambda}$, and $\mathrm{d}^{\Lambda}$, and the $\mathfrak{s l}(2 ; \mathbb{R})$-module generators $L, \Lambda$, and $H$, hold straightforwardly, [29, Lemma 2.3]:

$$
\begin{aligned}
& {[\mathrm{d}, L]=0,\left[\mathrm{~d}^{\Lambda}, L\right]=-\mathrm{d},\left[\mathrm{d} \mathrm{d}^{\Lambda}, L\right]=0,} \\
& {[\mathrm{~d}, \Lambda]=: \mathrm{d}^{\Lambda},\left[\mathrm{d}^{\Lambda}, \Lambda\right]=0,\left[\mathrm{~d} \mathrm{~d}^{\Lambda}, \Lambda\right]=0,} \\
& {[\mathrm{~d}, H]=\mathrm{d},\left[\mathrm{d}^{\Lambda}, H\right]=-\mathrm{d}^{\Lambda},\left[\mathrm{d} \mathrm{d}^{\Lambda}, H\right]=0 .}
\end{aligned}
$$

Hence, setting

$$
\mathrm{P} H_{\mathrm{d}+\mathrm{d}^{\Lambda}}^{\bullet}(X ; \mathbb{R}):=\frac{\operatorname{kerd} \cap \operatorname{ker} \mathrm{d}^{\Lambda} \cap \mathrm{P} \wedge^{\bullet} X}{\left.\operatorname{imdd^{\Lambda }\cap \mathrm {P}\wedge ^{\bullet }X}=\frac{\operatorname{kerd} \cap \mathrm{P} \wedge^{\bullet} X}{\mathrm{dd}^{\Lambda}(\mathrm{P} \wedge} \wedge^{\bullet} X\right)}
$$

(see [29, Lemma 3.9]), one gets that

$$
H_{\mathrm{d}+\mathrm{d}^{\Lambda}}^{\bullet}(X ; \mathbb{R})=\bigoplus_{r \in \mathbb{N}} L^{r} \mathrm{P} H_{\mathrm{d}+\mathrm{d}^{\Lambda}}^{\bullet-2 r}(X ; \mathbb{R})
$$

and, for every $k \in \mathbb{N}$,

$$
L^{k}: H_{\mathrm{d}+\mathrm{d}^{\Lambda}}^{n-k}(X ; \mathbb{R}) \stackrel{\simeq}{\longrightarrow} H_{\mathrm{d}+\mathrm{d}^{\Lambda}}^{n+k}(X ; \mathbb{R}),
$$

see [29, Theorem 3.11]. 
1.3. Hard Lefschetz condition. The identity map induces the following natural maps in cohomology:

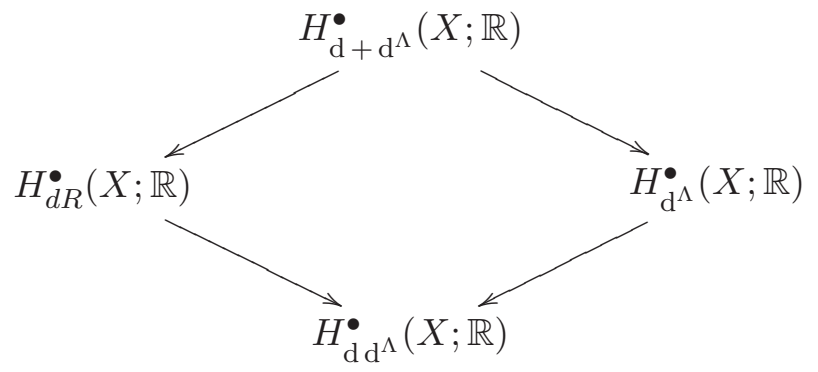

Recall that a symplectic manifold is said to satisfy the $\mathrm{d}^{\Lambda}-L e m m a$ if every $\mathrm{d}$-exact, $\mathrm{d}^{\Lambda}$-closed form is $\mathrm{d} \mathrm{d}^{\Lambda}$-exact, namely, if $H_{\mathrm{d}+\mathrm{d}^{\Lambda}}^{\bullet}(X ; \mathbb{R}) \rightarrow$ $H_{d R}^{\bullet}(X ; \mathbb{R})$ is injective. Furthermore, one says that the Hard Lefschetz Condition holds on $X$ if

$(\mathrm{HLC}) \quad$ for every $k \in \mathbb{N}, \quad L^{k}: H_{d R}^{n-k}(X ; \mathbb{R}) \stackrel{\simeq}{\longrightarrow} H_{d R}^{n+k}(X ; \mathbb{R})$.

In fact, by [24, Corollary 2], [32, Theorem 0.1], [25, Proposition 1.4], [18], [9, Theorem 5.4], (compare also [13]), it turns out that the following conditions are equivalent:

- $X$ satisfies the $\mathrm{d} \mathrm{d}^{\Lambda}$-Lemma;

- the natural homomorphism $H_{\mathrm{d}+\mathrm{d}^{\Lambda}}^{\bullet}(X ; \mathbb{R}) \rightarrow H_{d R}^{\bullet}(X ; \mathbb{R})$ is actually an isomorphism;

- every de Rham cohomology class admits a representative being both d-closed and $\mathrm{d}^{\Lambda}$-closed (i.e., Brylinski's conjecture [8, Conjecture 2.2.7] holds on $X$ );

- the Hard Lefschetz Condition holds on $X$.

1.4. Primitive currents. Denote by $\mathcal{D} \cdot X=\mathcal{D}^{2 n-\bullet} X$ the space of currents (i.e., the topological dual space of $\wedge^{\bullet} X$, endowed with the usual topology, see, e.g., [14, Section 9]). The differential d: $\mathcal{D} \bullet X \rightarrow \mathcal{D}_{\bullet-1} X$ is defined by duality from $\mathrm{d}: \wedge^{\bullet-1} X \rightarrow \wedge^{\bullet} X$. Hence, one can consider the de Rham homology $H_{\bullet}^{d R}(X ; \mathbb{R})$ as the homology of the complex $(\mathcal{D} \bullet X, \mathrm{~d})$. One has a natural injective homomorphism given by

$$
\begin{aligned}
T: \wedge^{\bullet} X & \rightarrow \mathcal{D}^{\bullet} X \\
\alpha & \mapsto T_{\alpha}:=\int_{X} \alpha \wedge ;
\end{aligned}
$$

since $\mathrm{d} \circ T=T \circ \mathrm{d}$, the homomorphism $T$ induces a map

$$
T: H_{d R}^{\bullet}(X ; \mathbb{R}) \rightarrow H_{\bullet}^{d R}(X ; \mathbb{R}) .
$$


Moreover, see [14, Theorem 14], one has an isomorphism

$$
H_{d R}^{\bullet}(X ; \mathbb{R}) \simeq H_{\bullet}^{d R}(X ; \mathbb{R}) ;
$$

in particular, $T: H_{d R}^{\bullet}(X ; \mathbb{R}) \rightarrow H_{\bullet}^{d R}(X ; \mathbb{R})$ is an isomorphism.

Following [22, Definition 4.1], set, by duality,

$$
\begin{aligned}
& L: \mathcal{D} \bullet X \rightarrow \mathcal{D}_{\bullet-2} X, S \mapsto S \circ L, \\
& \Lambda: \mathcal{D} \bullet X \rightarrow \mathcal{D}_{\bullet+2} X, \quad S \mapsto S \circ \Lambda, \\
& H: \mathcal{D} \bullet X \rightarrow \mathcal{D} \bullet X, \quad S \mapsto S \circ(-H),
\end{aligned}
$$

A current $S \in \mathcal{D}^{k} X$ is said to be primitive if $\Lambda S=0$, or, equivalently, if $L^{n-k+1} S=0$ (see, e.g., [22, Proposition 4.3]); denote by $\mathrm{PD}^{\bullet} X=\mathrm{PD}_{2 n-\bullet} X$ the space of primitive currents on $X$.

The following results are proven by Lin in [22] and provide a Lefschetz decomposition also on the space of currents, more precisely, on the space of compactly supported currents on a possibly non-compact symplectic manifold.

Proposition 1.1 ([22, Lemma 4.2]). Let $X$ be a closed manifold endowed with a symplectic structure. Then $\langle L, \Lambda, H\rangle$ gives a $\mathfrak{s l}(2 ; \mathbb{R})$-module structure on $\mathcal{D}^{\bullet} X$.

In particular, we get a Lefschetz decomposition on the space of currents, $[\mathbf{2 2}$, Proposition 4.3]:

$$
\mathcal{D}^{\bullet} X=\bigoplus_{r \in \mathbb{N}} L^{r} \mathrm{P} \mathcal{D}^{\bullet-2 r} X:=\bigoplus_{r \in \mathbb{N}} L^{r} \mathrm{PD}_{2 n-\bullet+2 r} X .
$$

Finally, if $j: Y \hookrightarrow X$ is a compact oriented submanifold of $X$ of codimension $k$, then the dual current $\rho_{Y} \in \mathcal{D}_{k} X$ associated with $Y$ is defined, by setting

$$
\rho_{Y}(\varphi):=\int_{Y} j^{*}(\varphi)
$$

for every test form $\varphi \in \wedge^{k} X$. If $Y$ is a closed oriented submanifold, then the dual current $\rho_{Y}$ is closed, and, according to $\left[\mathbf{2 9}\right.$, Lemma 4.1], $\rho_{Y}$ is primitive if and only if $Y$ is co-isotropic.

\section{Symplectic (co)homology decomposition}

In this section, we provide a symplectic counterpart to Li and Zhang's theory on cohomology of almost-complex manifolds developed in [21].

Let $X$ be a $2 n$-dimensional closed manifold endowed with a symplectic structure $\omega$. For any $r, s \in \mathbb{N}$, define

$$
H_{\omega}^{(r, s)}(X ; \mathbb{R}):=\left\{\left[L^{r} \beta^{(s)}\right] \in H_{d R}^{2 r+s}(X ; \mathbb{R}): \beta^{(s)} \in \mathrm{P} \wedge^{s} X\right\} \subseteq H_{d R}^{2 r+s}(X ; \mathbb{R})
$$


Obviously, for every $k \in \mathbb{N}$, one has

$$
\sum_{2 r+s=k} H_{\omega}^{(r, s)}(X ; \mathbb{R}) \subseteq H_{d R}^{k}(X ; \mathbb{R}) ;
$$

we are concerned in studying when the above inclusion is actually an equality, and when the sum is actually a direct sum.

Remark 2.1. We underline the relations between the above subgroups and the primitive cohomologies introduced by Tseng and Yau in [29].

As regards Tseng and Yau's primitive $\left(\mathrm{d}+\mathrm{d}^{\Lambda}\right)$-cohomology $\mathrm{P}_{\mathrm{d}+\mathrm{d}^{\Lambda}}^{\bullet}(X ; \mathbb{R})$, note that, for every $r, s \in \mathbb{N}$,

$$
\operatorname{im}\left(L^{r}: \mathrm{P} H_{\mathrm{d}+\mathrm{d}^{\Lambda}}^{s}(X ; \mathbb{R}) \rightarrow H_{d R}^{\bullet}(X ; \mathbb{R})\right)=L^{r} H_{\omega}^{(0, s)}(X ; \mathbb{R}) \subseteq H_{\omega}^{(r, s)}(X ; \mathbb{R}) .
$$

In [29, Section 4.1], Tseng and Yau have introduced also the primitive cohomology groups

$$
\mathrm{P} H_{\mathrm{d}}^{s}(X ; \mathbb{R}):=\frac{\operatorname{kerd} \cap \operatorname{ker} \mathrm{d}^{\Lambda} \cap \mathrm{P} \wedge^{s} X}{\operatorname{imd}\left\lfloor_{\mathrm{P} \wedge^{s-1} X \cap \operatorname{ker~}^{\Lambda}}\right.},
$$

where $s \in \mathbb{N}$, proving that the homology on co-isotropic chains is naturally dual to $\mathrm{P}_{\mathrm{d}}^{2 n-\bullet}(X ; \mathbb{R})$, see [29, page 41]; in [22, Proposition A.5], Lin has proved that, if the Hard Lefschetz Condition holds on $X$, then

$$
H_{\omega}^{(0, \bullet)}(X ; \mathbb{R})=\mathrm{P} H_{\mathrm{d}}^{\bullet}(X ; \mathbb{R}) .
$$

Remark 2.2. In [11], Conti and the second author studied the notion of half-flat structure on a six-dimensional manifold $X$ (see [10]). Namely, an $\mathrm{SU}(3)$-structure $(\omega, \psi)$ on $X$ (where $\omega$ is a non-degenerate real 2-form, and $\psi$ is a decomposable complex 3 -form, such that $\psi \wedge \omega=0$ and $\psi \wedge \bar{\psi}=-\frac{4 \mathrm{i}}{3} \omega^{3}$ ) is called half-flat if both $\omega \wedge \omega$ and $\Re \mathfrak{e} \psi$ are d-closed. Note in particular that, if $(\omega, \psi)$ is a symplectic half-flat structure on $X$, then $[\Re \mathfrak{e} \psi] \in H_{\omega}^{(0,3)}(X ; \mathbb{R})$. Furthermore, $\Re \mathfrak{e} \psi$ is a calibration on $X$ and special Lagrangian submanifolds are naturally defined also in this context.

Remark 2.3. A class of example of closed symplectic manifolds satisfying the cohomology decomposition by means of the above subgroups $H_{\omega}^{\bullet, \bullet}(X ; \mathbb{R})$ (actually, satisfying an even stronger cohomology decomposition) is provided by the closed symplectic manifolds satisfying the $\mathrm{d} \mathrm{d}^{\Lambda}$-Lemma, equivalently, the Hard Lefschetz Condition. More precisely, recall that, by [32] (see also [29, Theorem 3.11, Proposition 3.13]), for a $2 n$-dimensional closed manifold $X$ endowed with a symplectic structure $\omega$, the following conditions are equivalent:

- $X$ satisfies the $\mathrm{d} \mathrm{d}^{\Lambda}$-Lemma;

- it holds that

$$
H_{d R}^{\bullet}(X ; \mathbb{R})=\bigoplus_{r \in \mathbb{N}} L^{r} H_{\omega}^{(0, \bullet-2 r)}(X ; \mathbb{R}) .
$$


Analogously, considering the space $\mathcal{D}^{\bullet} X$ of currents and the de Rham homology $H_{\bullet}^{d R}(X ; \mathbb{R})$, for every $r, s \in \mathbb{N}$, define

$$
\begin{aligned}
H_{(r, s)}^{\omega}(X ; \mathbb{R}):= & \left\{\left[L^{r} B_{(s)}\right] \in H_{-2 r+s}^{d R}(X ; \mathbb{R}): B_{(s)} \in \mathrm{PD}_{s} X\right\} \\
& \subseteq H_{-2 r+s}^{d R}(X ; \mathbb{R}) ;
\end{aligned}
$$

as previously, for every $k \in \mathbb{N}$, we have just the inclusion

$$
\sum_{-2 r+s=k} H_{(r, s)}^{\omega}(X ; \mathbb{R}) \subseteq H_{k}^{d R}(X ; \mathbb{R}),
$$

but, in general, neither the sum is direct nor the inclusion is an equality.

We prove that, fixed $k \in \mathbb{N}$, if the sum $\sum_{2 r+s=2 n-k} H_{\omega}^{(r, s)}(X ; \mathbb{R})$ gives the whole $(2 n-k)^{\text {th }}$ de Rham cohomology group, then the sum of the subgroups of the $k^{\text {th }}$ de Rham cohomology group is direct (this result should be compared with [21, Proposition 2.30], see also [2, Theorem 2.1], in the almost-complex case).

Proposition 2.4. Let $X$ be a $2 n$-dimensional closed manifold endowed with a symplectic structure $\omega$. For every $k \in \mathbb{N}$, the following implications hold:

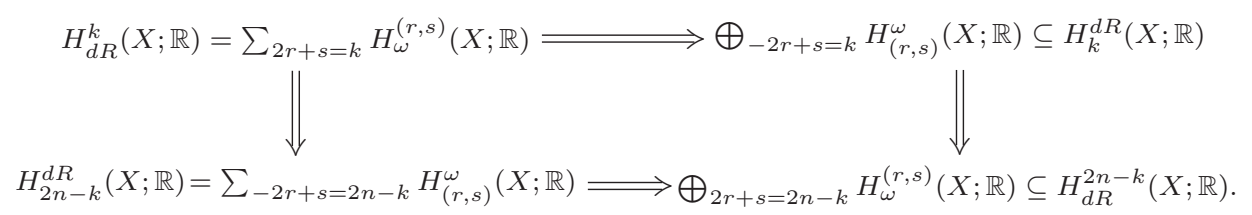

Proof. Note that the quasi-isomorphism $T: \wedge^{\bullet} X \rightarrow \mathcal{D}^{\bullet} X$ satisfies

$$
T \circ L=L \circ T \text {, }
$$

and hence, in particular, it preserves the bi-graduation,

$$
T\left(L^{\bullet} \mathrm{P} \wedge^{\bullet} X\right) \subseteq L^{\bullet} \mathrm{P} \mathcal{D}^{\bullet} X=L^{\bullet} \mathrm{PD}_{2 n-\bullet} X,
$$

and it induces, for every $r, s \in \mathbb{N}$, an injective map

$$
H_{\omega}^{(r, s)}(X ; \mathbb{R}) \hookrightarrow H_{(r, 2 n-s)}^{\omega}(X ; \mathbb{R}) .
$$

Therefore, the two vertical implications are proven.

Consider now the non-degenerate duality pairing

$$
\langle\cdot, \cdot \cdot\rangle: H_{d R}^{\bullet}(X ; \mathbb{R}) \times H_{\bullet}^{d R}(X ; \mathbb{R}) \rightarrow \mathbb{R},
$$

and note that, for every $r, s \in \mathbb{N}$,

$$
\operatorname{ker}\left\langle H_{\omega}^{(r, s)}(X ; \mathbb{R}), \cdot\right\rangle \supseteq \sum_{(p, q) \neq(n-r-s, 2 n-s)} H_{(p, q)}^{\omega}(X ; \mathbb{R}) ;
$$

indeed, recall that, for any $k \in \mathbb{N}$, it holds

$$
\mathrm{P} \wedge^{k} X:=\operatorname{ker} \Lambda\left\lfloor_{\wedge^{k} X}=\operatorname{ker} L^{n-k+1}\left\lfloor_{\wedge^{k} X}\right.\right.
$$


and

$$
\mathrm{PD}^{k} X=\mathrm{PD}_{2 n-k} X:=\operatorname{ker} \Lambda\left\lfloor_{\mathcal{D}^{k} X}=\operatorname{ker} L^{n-k+1}\left\lfloor_{\mathcal{D}^{k} X},\right.\right.
$$

and that, if $2 r+s \neq-2 p+q$, then

$$
\left\langle H_{\omega}^{(r, s)}(X ; \mathbb{R}), H_{(p, q)}^{\omega}(X ; \mathbb{R})\right\rangle=\{0\} .
$$

Arguing in the same way in the case of currents, this suffices to prove the two horizontal implications.

A straightforward consequence of [24, Corollary 2], or [32, Theorem 0.1], and Proposition 2.4 is the following result, which should be compared with [15, Theorem 2.16, Proposition 2.17].

Corollary 2.5. Let $X$ be a closed manifold endowed with a symplectic structure $\omega$. Suppose that the Hard Lefschetz Condition holds on X, equivalently, that $X$ satisfies the $\mathrm{d}^{\Lambda}$-Lemma. Then

$$
H_{d R}^{\bullet}(X ; \mathbb{R})=\bigoplus_{r \in \mathbb{N}} H_{\omega}^{(r, \bullet-2 r)}(X ; \mathbb{R})
$$

and

$$
H_{\bullet}^{d R}(X ; \mathbb{R})=\bigoplus_{r \in \mathbb{N}} H_{(r, \bullet+2 r)}^{\omega}(X ; \mathbb{R})
$$

In particular, when $\operatorname{dim} X=4$ and taking $k=2$ in Proposition 2.4, one gets that, if $H_{d R}^{2}(X ; \mathbb{R})=H_{\omega}^{(1,0)}(X ; \mathbb{R})+H_{\omega}^{(0,2)}(X ; \mathbb{R})$ holds, then actually $H_{d R}^{2}(X ; \mathbb{R})=H_{\omega}^{(1,0)}(X ; \mathbb{R}) \oplus H_{\omega}^{(0,2)}(X ; \mathbb{R})$ holds. In fact, the following result states that $H_{d R}^{2}(X ; \mathbb{R})$ always decomposes as direct sum of $H_{\omega}^{(1,0)}(X ; \mathbb{R})$ and $H_{\omega}^{(0,2)}(X ; \mathbb{R})$, also in dimension higher than 4 : this gives a symplectic counterpart to Drăghici et al. [15, Theorem 2.3] in the complex setting (in fact, without the restriction to dimension 4).

Theorem 2.6. Let $X$ be a closed manifold endowed with a symplectic structure $\omega$. Then

$$
H_{d R}^{2}(X ; \mathbb{R})=H_{\omega}^{(1,0)}(X ; \mathbb{R}) \oplus H_{\omega}^{(0,2)}(X ; \mathbb{R}) .
$$

In particular, if $\operatorname{dim} X=4$, then

$$
H_{d R}^{\bullet}(X ; \mathbb{R})=\bigoplus_{r \in \mathbb{N}} H_{\omega}^{(r, \bullet-2 r)}(X ; \mathbb{R})
$$

and

$$
H_{\bullet}^{d R}(X ; \mathbb{R})=\bigoplus_{r \in \mathbb{N}} H_{(r, \bullet+2 r)}^{\omega}(X ; \mathbb{R})
$$


Proof. Firstly, we prove that $H_{\omega}^{(1,0)}(X ; \mathbb{R}) \cap H_{\omega}^{(0,2)}(X ; \mathbb{R})=\{0\}$. Let

$$
\mathfrak{c}=[f \omega]=\left[\beta^{(2)}\right] \in H_{\omega}^{(1,0)}(X ; \mathbb{R}) \cap H_{\omega}^{(0,2)}(X ; \mathbb{R}),
$$

where $f \in \mathcal{C}^{\infty}(X ; \mathbb{R})$ and $\beta^{(2)} \in \mathrm{P} \wedge^{2} X$. Being $\mathrm{P} \wedge^{2} X=\operatorname{ker} L^{n-1} \iota_{\wedge^{2} X}$, one has

$$
\begin{aligned}
0 & =\int_{X} f L^{n-1} \beta^{(2)}=\int_{X} f \omega \wedge \beta^{(2)} \wedge \omega^{n-2} \\
& =\int_{X} f \omega \wedge f \omega \wedge \omega^{n-2}=\int_{X} f^{2} \omega^{n},
\end{aligned}
$$

hence $f=0$, that is, $\mathfrak{c}=0$.

Now, we prove that $H_{d R}^{2}(X ; \mathbb{R})=H_{\omega}^{(1,0)}(X ; \mathbb{R})+H_{\omega}^{(0,2)}(X ; \mathbb{R})$. Let $2 n$ be the dimension of $X$. Note that, for any $[\alpha] \in H_{d R}^{2}(X ; \mathbb{R})$, for dimensional reasons, one has $L^{n-1}[\alpha]=\lambda\left[\omega^{n}\right]$ for some $\lambda \in \mathbb{R}$. Thus $L^{n-1}([\alpha]-\lambda[\omega])=0$ in $H_{d R}^{2 n}(X ; \mathbb{R})$. It follows that there exists $\beta \in \wedge^{2 n-1} X$ such that $L^{n-1}(\alpha-\lambda \omega)=\mathrm{d} \beta$. Since $L^{n-1}: \wedge^{1} X \rightarrow \wedge^{2 n-1} X$ is an isomorphism, there exists $\gamma \in \wedge^{1} X$ such that $\beta=L^{n-1} \gamma$. Hence, one gets that $L^{n-1}(\alpha-\lambda \omega-\mathrm{d} \gamma)=0$, that is, $[\alpha-\mathrm{d} \gamma]-\lambda[\omega] \in H_{\omega}^{(0,2)}(X ; \mathbb{R})$, completing the proof.

Remark 2.7. Note that the argument in the proof of Theorem 2.6 can be generalized to prove the following:

If $X$ is a $2 n$-dimensional closed manifold endowed with a symplectic structure $\omega$, then, for every $k \in\left\{1, \ldots,\left\lfloor\frac{n}{2}\right\rfloor\right\}$, it holds

$$
H_{\omega}^{(k, 0)}(X ; \mathbb{R}) \cap H_{\omega}^{(0,2 k)}(X ; \mathbb{R})=\{0\} .
$$

In some cases, the study of the spaces $H_{\omega}^{(r, s)}(X ; \mathbb{R})$ can be reduced to the study of $H_{\omega}^{(0, r)}(X ; \mathbb{R})$ : this is the matter of the following result.

Proposition 2.8. Let $X$ be a $2 n$-dimensional closed manifold endowed with a symplectic structure $\omega$. Then, for every $r, s \in \mathbb{N}$ such that $2 r+s \leq n$, one has

$$
H_{\omega}^{(r, s)}(X ; \mathbb{R})=L^{r} H_{\omega}^{(0, s)}(X ; \mathbb{R}) .
$$

Proof. Since $L: \wedge^{j} X \rightarrow \wedge^{j+2} X$ is injective for $j \leq n-1$ (in fact, an isomorphism for $j=n-1)$, and $[\mathrm{d}, L]=0$, we get that

$$
\begin{aligned}
& H_{\omega}^{(r, s)}(X ; \mathbb{R}) \\
& \quad=\left\{\left[\omega^{r} \beta^{(s)}\right] \in H_{d R}^{2 r+s}(X ; \mathbb{R}): \beta^{(s)} \in \wedge^{s} X \cap \operatorname{ker} \Lambda \text { such that } L^{r} \mathrm{~d} \beta^{(s)}=0\right\} \\
& \quad=\left\{\left[\omega^{r}\right] \smile\left[\beta^{(s)}\right] \in H_{d R}^{2 r+2}(X ; \mathbb{R}): \beta^{(s)} \in \wedge^{s} X \cap \operatorname{ker} \Lambda\right\},
\end{aligned}
$$

assumed that $2 r+s \leq n$. 
In particular, for every $r \in\left\{1, \ldots,\left\lfloor\frac{n}{2}\right\rfloor\right\}$, the spaces $H_{\omega}^{(r, 0)}(X ; \mathbb{R})$ are onedimensional $\mathbb{R}$-vector spaces: more precisely, $H_{\omega}^{(r, 0)}(X ; \mathbb{R})=\mathbb{R}\left\langle\left[\omega^{r}\right]\right\rangle$.

In particular, by the previous result follows that, for $k \leq n$, the condition

$$
H_{d R}^{k}(X ; \mathbb{R})=\bigoplus_{r \in \mathbb{N}} H_{\omega}^{(r, k-2 r)}(X ; \mathbb{R})
$$

is in fact equivalent to $H_{d R}^{k}(X ; \mathbb{R})=\bigoplus_{r \in \mathbb{N}} L^{r} H_{\omega}^{(0, k-2 r)}(X ; \mathbb{R})$.

\section{Symplectic (co)homology decomposition on solvmanifolds}

By a nilmanifold (respectively, a solvmanifold) we mean a compact quotient of a nilpotent (respectively, solvable) Lie group by a discrete co-compact subgroup. A solvmanifold $X=\Gamma \backslash G$ is called completely solvable if, for any $g \in G$, all the eigenvalues of $\operatorname{Ad} g \in \operatorname{End}(G)$ are real, equivalently, if, for any $X \in \mathfrak{g}$, all the eigenvalues of $\operatorname{ad} X \in \operatorname{End}(\mathfrak{g})$ are real.

To shorten the notation, we will refer to a given solvmanifold $X=\Gamma \backslash G$ by means of the structure equations of its Lie algebra $\mathfrak{g}$; for example, by writing

$$
X:=\left(0^{4}, 12,13\right)
$$

we mean that there exists a basis $\left\{e^{1}, \ldots, e^{6}\right\}$ of $\mathfrak{g}^{*}$ with respect to which the structure equations are

$$
\left\{\begin{array}{l}
\mathrm{d} e^{1}=\mathrm{d} e^{2}=\mathrm{d} e^{3}=\mathrm{d} e^{4}=0 \\
\mathrm{~d} e^{5}=e^{1} \wedge e^{2}=: e^{12} \\
\mathrm{~d} e^{6}=e^{1} \wedge e^{3}=: e^{13}
\end{array}\right.
$$

where we shorten $e^{A B}:=e^{A} \wedge e^{B}$. Recall that, by Mal'tsev's theorem [23, Theorem 7], given a nilpotent Lie algebra $\mathfrak{g}$ with rational structure constants, then the connected simply-connected Lie group $G$ naturally associated to $\mathfrak{g}$ admits a co-compact discrete subgroup $\Gamma$, and hence there exists a nilmanifold $X:=\Gamma \backslash G$ whose Lie algebra is $\mathfrak{g}$. Dealing with $G$-left-invariant objects on $X$, we mean objects induced by objects on $G$ which are invariant under the left-action of $G$ on itself given by left-translations. By means of left-translations, $G$-left-invariant objects will be identified with objects on the Lie algebra.

By Hattori's theorem [19, Corollary 4.2], the cohomology of a completely solvable solvmanifold $X$ is isomorphic to the cohomology $H^{\bullet}(\mathfrak{g} ; \mathbb{R}):=$ $H^{\bullet}\left(\wedge^{\bullet} \mathfrak{g}^{*}, \mathrm{~d}\right)$ of the complex $\left(\wedge^{\bullet} \mathfrak{g}^{*}, \mathrm{~d}\right)$, where $\mathrm{d}: \wedge^{\bullet} \mathfrak{g}^{*} \rightarrow \wedge^{\bullet+1} \mathfrak{g}^{*}$ is induced by $\mathrm{d}_{\mathfrak{g}}: \wedge^{1} \mathfrak{g}^{*} \rightarrow \wedge^{2} \mathfrak{g}^{*},\left(\mathrm{~d}_{\mathfrak{g}} \alpha\right)(x, y):=-\alpha([x, y])$ : for simplicity, in writing the cohomology of a solvmanifold, we list the harmonic representatives with respect to the $G$-left-invariant metric $g:=\sum_{\ell} e^{\ell} \odot e^{\ell}$ instead of their classes. 
We recall that, by Benson and Gordon's theorem [6, Theorem A], if a nilmanifold $X$ is endowed with a symplectic structure $\omega$ such that the Hard Lefschetz Condition holds, then it is diffeomorphic to a torus.

Let $X=\Gamma \backslash G$ be a completely solvable solvmanifold, endowed with a $G$ left-invariant structure $\omega$. In particular, $\omega$ being $G$-left-invariant, $\langle L, \Lambda, H\rangle$ induces a $\mathfrak{s l}(2 ; \mathbb{R})$-representation both on $\wedge^{\bullet} X$ and on its (quasi-isomorphic) subspace made of the $G$-left-invariant forms (which is isomorphic to $\wedge^{\bullet} \mathfrak{g}^{*}$ ). For any $r, s \in \mathbb{N}$, we can consider both the subgroup $H_{\omega}^{(r, s)}(X ; \mathbb{R})$ of $H_{d R}^{\bullet}(X ; \mathbb{R})$, and the subgroup

$$
H_{\omega}^{(r, s)}(\mathfrak{g} ; \mathbb{R}):=\left\{\left[L^{r} \beta^{(s)}\right] \in H^{\bullet}(\mathfrak{g} ; \mathbb{R}): \Lambda \beta^{(s)}=0\right\}
$$

of $H^{\bullet}(\mathfrak{g} ; \mathbb{R}) \simeq H_{d R}^{\bullet}(X ; \mathbb{R})$, namely, the subgroup made of the de Rham cohomology classes admitting $G$-left-invariant representatives in $L^{r} \mathrm{P} \wedge{ }^{s} X$.

In this section, we are concerned in studying the linking between $H_{\omega}^{(\bullet, \bullet)}(X ; \mathbb{R})$ and $H_{\omega}^{(\bullet, \bullet)}(\mathfrak{g} ; \mathbb{R})$. This will let us study explicit examples.

First of all, we will need the following lemma by Milnor.

Lemma 3.1 ([26, Lemma 6.2]). Any connected Lie group that admits a discrete subgroup with compact quotient is unimodular and in particular admits a bi-invariant volume form $\eta$.

In the following lemma, we recall Belgun's symmetrization trick; see [5, Theorem 7] and [16, Theorem 2.1].

Lemma 3.2. Let $X=\Gamma \backslash G$ be a solvmanifold and call $\mathfrak{g}$ the Lie algebra naturally associated to the connected simply-connected Lie group G. Let $\omega$ be a $G$-left-invariant symplectic structure on $X$. Let $\eta$ be the $G$-bi-invariant volume form on $G$ given by Milnor's Lemma 3.1 and such that $\int_{X} \eta=1$. (Up to identifying $G$-left-invariant forms with linear forms over $\mathfrak{g}^{*}$ through left-translations,) define the map

$$
\mu: \wedge^{k} X \rightarrow \wedge^{k} \mathfrak{g}^{*}, \quad \mu(\alpha)\left(X_{1}, \ldots, X_{k}\right):=\int_{X} \alpha_{m}\left(\left.X_{1}\right|_{m}, \ldots,\left.X_{k}\right|_{m}\right) \eta_{m} .
$$

One has that

$$
\mu\left\lfloor_{\wedge} \mathfrak{g}^{*}=\operatorname{id}_{\wedge} \bullet \mathfrak{g}^{*}: \wedge^{\bullet} \mathfrak{g}^{*} \rightarrow \wedge^{\bullet} \mathfrak{g}^{*} \subseteq \wedge^{\bullet} X\right.
$$

and that

$$
\sharp \circ \mu=\mu \circ \sharp, \quad \text { for } \quad \sharp \in\{\mathrm{d}, L\} .
$$

In particular, $\mu$ sends primitive forms to $G$-left-invariant primitive forms.

Proof. It has to be shown just that $\mu(L \alpha)=L \mu(\alpha)$ for every $\alpha \in$ $\wedge \cdot X$. Note that, $\omega$ being a G-left-invariant form, one has $\mu(L \alpha)=$ $\int_{X}(\omega \wedge \alpha)_{m} \eta_{m}=\int_{X} \omega_{m} \wedge \alpha_{m} \eta_{m}=\omega \wedge \int_{X} \alpha_{m} \eta_{m}=L \mu(\alpha)$. 
Then we can prove the following result, which relates the subgroups $H_{\omega}^{(r, s)}(X ; \mathbb{R})$ with their invariant part $H_{\omega}^{(r, s)}(\mathfrak{g} ; \mathbb{R})$ (compare it with [1, Proposition 2.4] for almost-D-complex structures in the sense of Harvey and Lawson, and also with [17, Theorem 3.4] for almost-complex structures).

Proposition 3.3. Let $X=\Gamma \backslash G$ be a solvmanifold endowed with a $G$-leftinvariant symplectic structure $\omega$. Call $\mathfrak{g}$ the Lie algebra naturally associated to the connected simply-connected Lie group $G$. For every $r, s \in \mathbb{N}$, the map

$$
j: H_{\omega}^{(r, s)}(\mathfrak{g} ; \mathbb{R}) \rightarrow H_{\omega}^{(r, s)}(X ; \mathbb{R})
$$

induced by left-translations is injective, and, if $H^{\bullet}(\mathfrak{g} ; \mathbb{R}) \simeq H_{d R}^{\bullet}(X ; \mathbb{R})$ (for instance, if $X$ is a completely solvable solvmanifold), then it is in fact an isomorphism.

Proof. Left-translations induce the map $j: H_{\omega}^{(r, s)}(\mathfrak{g} ; \mathbb{R}) \rightarrow H_{\omega}^{(r, s)}(X ; \mathbb{R})$. Consider the Belgun's symmetrization map $\mu: \wedge^{\bullet} X \rightarrow \wedge^{\bullet} \mathfrak{g}^{*}$ : by Lemma 3.2, since it commutes with $\mathrm{d}$, it induces the map $\mu: H_{d R}^{\bullet}(X ; \mathbb{R}) \rightarrow H^{\bullet}(\mathfrak{g} ; \mathbb{R})$, and, since it commutes with $L$ and $\Lambda$, it induces the map $\mu: H_{\omega}^{(r, s)}(X ; \mathbb{R}) \rightarrow$ $H_{\omega}^{(r, s)}(\mathfrak{g} ; \mathbb{R})$. Moreover, since $\mu$ is the identity on the space of $G$-left-invariant forms, we get the commutative diagram

$$
H_{\omega}^{(r, s)}(\mathfrak{g} ; \mathbb{R}) \underbrace{\stackrel{j}{\longrightarrow} H_{\omega}^{(r, s)}(X ; \mathbb{R}) \stackrel{\mu}{\longrightarrow}}_{\text {id }} H_{\omega}^{(r, s)}(\mathfrak{g} ; \mathbb{R})
$$

Hence, $j: H_{\omega}^{(r, s)}(\mathfrak{g} ; \mathbb{R}) \rightarrow H_{\omega}^{(r, s)}(X ; \mathbb{R})$ is injective, and $\mu: H_{\omega}^{(r, s)}(X ; \mathbb{R}) \rightarrow$ $H_{\omega}^{(r, s)}(\mathfrak{g} ; \mathbb{R})$ is surjective.

Furthermore, when $H^{\bullet}(\mathfrak{g} ; \mathbb{R}) \simeq H_{d R}^{\bullet}(X ; \mathbb{R})$ (for instance, when $X$ is a completely solvable solvmanifold, by Hattori's theorem [19, Theorem 4.2]), since $\mu\left\lfloor_{\wedge} \mathfrak{g}^{*}=\mathrm{id}\left\lfloor_{\wedge} \mathfrak{g}^{*}\right.\right.$, we get that $\mu: H_{d R}^{\bullet}(X ; \mathbb{R}) \rightarrow H^{\bullet}(\mathfrak{g} ; \mathbb{R})$ is the identity map, and hence $\mu: H_{\omega}^{(r, s)}(X ; \mathbb{R}) \rightarrow H_{\omega}^{(r, s)}(\mathfrak{g} ; \mathbb{R})$ is also injective, hence an isomorphism.

Proposition 3.3 is a useful tool to study explicit examples.

Example 3.4. Take the six-dimensional nilmanifold

$$
X:=\left(0^{3}, 12,14-23,15+34\right)
$$

endowed with the left-invariant symplectic structure

$$
\omega:=e^{16}+e^{35}+e^{24} .
$$


By Nomizu's theorem [28, Theorem 1], one computes

$$
\begin{aligned}
& H_{d R}^{1}(X ; \mathbb{R})=\underbrace{\mathbb{R}\left\langle e^{1}, e^{2}, e^{3}\right\rangle}_{=H_{\omega}^{(0,1)}(X ; \mathbb{R})}, \\
& H_{d R}^{2}(X ; \mathbb{R})=\underbrace{\mathbb{R}\left\langle e^{16}+e^{35}+e^{24}\right\rangle}_{=H_{\omega}^{(1,0)}(X ; \mathbb{R})} \oplus \underbrace{\mathbb{R}\left\langle e^{13}, e^{14}+e^{23}, 2 \cdot e^{24}-e^{16}-e^{35}\right\rangle}_{=H_{\omega}^{(0,2)}(X ; \mathbb{R})}, \\
& H_{d R}^{3}(X ; \mathbb{R})=\mathbb{R}\left\langle e^{126}-e^{145}-2 \cdot e^{235}, e^{136}, e^{146}+\frac{1}{2} \cdot e^{236}+\frac{1}{2} \cdot e^{345}, e^{245}\right\rangle .
\end{aligned}
$$

Since the Lefschetz decompositions of the $g$-harmonic representatives of $H_{d R}^{3}(X ; \mathbb{R})$ are

$$
\begin{aligned}
e^{126}-e^{145}-2 \cdot e^{235}= & \underbrace{\left(-\frac{1}{2} \cdot e^{126}-\frac{1}{2} \cdot e^{235}-e^{145}\right)}_{\in \mathrm{P} \wedge^{3} X} \\
& +\underbrace{\left(\frac{3}{2} \cdot e^{126}-\frac{3}{2} \cdot e^{235}\right)}_{=L\left(-\frac{3}{2} \cdot e^{2}\right)}, \\
e^{136}= & \underbrace{\left(\frac{1}{2} \cdot e^{136}-\frac{1}{2} \cdot e^{234}\right)}_{\in \mathrm{P} \wedge^{3} X}+\underbrace{\left(\frac{1}{2} \cdot e^{136}+\frac{1}{2} \cdot e^{234}\right)}_{=L\left(-\frac{1}{2} \cdot e^{3}\right)}, \\
e^{146}+\frac{1}{2} \cdot e^{236}+\frac{1}{2} \cdot e^{345}= & \underbrace{\left(\frac{1}{4} \cdot e^{146}-\frac{1}{4} \cdot e^{345}+\frac{1}{2} \cdot e^{236}\right)}_{\in \mathrm{P} \wedge^{3} X} \\
& +\underbrace{\left(\frac{3}{4} \cdot e^{146}+\frac{3}{4} \cdot e^{345}\right)}_{=L\left(-\frac{3}{4} \cdot e^{4}\right)}, \underbrace{\left(-\frac{1}{2} \cdot e^{156}+\frac{1}{2} \cdot e^{245}\right)}_{=L\left(\frac{1}{2} \cdot e^{5}\right)}, \\
e^{245}= & \underbrace{\left(\frac{1}{2} \cdot e^{156}+\frac{1}{2} \cdot e^{245}\right)}_{\in \wedge^{3} X}+\underbrace{\left(-\frac{1}{2}\right)}
\end{aligned}
$$

and since

$\mathrm{d} \wedge^{2} \mathfrak{g}^{*}=\mathbb{R}\left\langle e^{123}, e^{124}, e^{125}, e^{126}+e^{145}, e^{134}, e^{135}, e^{146}-e^{236}-e^{345}, e^{234}\right\rangle$, 
we get that

$$
\begin{aligned}
{\left[e^{126}-e^{145}-2 \cdot e^{235}\right] } & =\left[e^{126}-e^{145}-2 \cdot e^{235}+\mathrm{d} e^{46}\right] \\
& =\left[2 \cdot e^{126}-2 \cdot e^{235}\right]=\left[L\left(-2 \cdot e^{2}\right)\right] \in H_{\omega}^{(1,1)}(X ; \mathbb{R})
\end{aligned}
$$

and

$$
\begin{aligned}
{\left[e^{136}\right]=} & {\left[e^{136}+\mathrm{d}\left(\frac{1}{2} \cdot e^{45}-\frac{1}{2} \cdot e^{26}\right)\right]=\left[e^{136}+e^{234}\right] } \\
& =\left[L\left(-e^{3}\right)\right] \in H_{\omega}^{(1,1)}(X ; \mathbb{R}) \\
{\left[e^{136}\right]=} & {\left[e^{136}-\mathrm{d}\left(\frac{1}{2} \cdot e^{45}-\frac{1}{2} \cdot e^{26}\right)\right]=\left[e^{136}-e^{234}\right] \in H_{\omega}^{(0,3)}(X ; \mathbb{R}), }
\end{aligned}
$$

while it is straightforward to check that

$\mathbb{R}\left\langle e^{146}+\frac{1}{2} \cdot e^{236}+\frac{1}{2} \cdot e^{345}, e^{245}\right\rangle \cap\left(H_{\omega}^{(0,3)}(X ; \mathbb{R})+H_{\omega}^{(1,1)}(X ; \mathbb{R})\right)=\{0\}$

in particular, $H_{\omega}^{(0,3)}(X ; \mathbb{R})+H_{\omega}^{(1,1)}(X ; \mathbb{R}) \subsetneq H_{d R}^{3}(X ; \mathbb{R})$ and $H_{\omega}^{(0,3)}(X ; \mathbb{R}) \cap$ $H_{\omega}^{(1,1)}(X ; \mathbb{R}) \neq\{0\}$.

Example 3.5. Take the six-dimensional solvable Lie algebra

$$
\mathfrak{g}_{3.4}^{-1} \oplus \mathfrak{g}_{3.5}^{0}:=(-13,23,0,-56,46,0)
$$

endowed with the linear symplectic structure

$$
\omega:=e^{12}+e^{36}+e^{45} .
$$

The corresponding connected simply connected Lie group admits a compact quotient, whose de Rham cohomology is the same as the cohomology of $\left(\wedge^{\bullet}\left(\mathfrak{g}_{3.4}^{-1} \oplus \mathfrak{g}_{3.5}^{0}\right)^{*}, \mathrm{~d}\right)$; see $[\mathbf{7}$, Table 5].

It is straightforward to compute

$$
\begin{aligned}
H_{d R}^{1}(X ; \mathbb{R})= & \underbrace{\mathbb{R}\left\langle e^{3}, e^{6}\right\rangle}_{=H_{\omega}^{(0,1)}(X ; \mathbb{R})}, \\
H_{d R}^{2}(X ; \mathbb{R})= & \underbrace{\mathbb{R}\left\langle e^{12}+e^{36}+e^{45}\right\rangle}_{=H_{\omega}^{(1,0)}(X ; \mathbb{R})} \oplus \underbrace{\mathbb{R}\left\langle e^{12}-e^{36}, e^{12}-e^{45}\right\rangle}_{=H_{\omega}^{(0,2)}(X ; \mathbb{R})},
\end{aligned}
$$




$$
\begin{aligned}
H_{d R}^{3}(X ; \mathbb{R}) & =\underbrace{\mathbb{R}\left\langle e^{123}+e^{345}, e^{126}+e^{456}\right\rangle}_{=H_{\omega}^{(1,1)}(X ; \mathbb{R})=L H_{\omega}^{(0,1)}(X ; \mathbb{R})} \oplus \underbrace{\mathbb{R}\left\langle e^{123}-e^{345}, e^{126}-e^{456}\right\rangle}_{=H_{\omega}^{(0,3)}(X ; \mathbb{R})}, \\
H_{d R}^{4}(X ; \mathbb{R})= & \underbrace{\mathbb{R}\left\langle e^{1236}+e^{1245}+e^{3456}\right\rangle}_{=H_{\omega}^{(2,0)}(X ; \mathbb{R})} \oplus \underbrace{\mathbb{R}\left\langle e^{1236}-e^{1245}, e^{1236}-e^{3456}\right\rangle}_{=H_{\omega}^{(1,2)}(X ; \mathbb{R})=L H_{\omega}^{(0,2)}(X ; \mathbb{R})}, \\
H_{d R}^{5}(X ; \mathbb{R})= & \underbrace{\mathbb{R}\left\langle e^{12456}, e^{12345}\right\rangle}_{\left(H_{\omega}^{(2,1)}(X ; \mathbb{R})=L^{2} H_{\omega}^{(0,1)}(X ; \mathbb{R})\right.},
\end{aligned}
$$

hence we get a decomposition

$$
H^{\bullet}(X ; \mathbb{R})=\bigoplus_{r \in \mathbb{N}} L^{r} H_{\omega}^{(0, \bullet-2 r)}(X ; \mathbb{R}) .
$$

In particular, it follows that the Hard Lefschetz Condition holds on $(X, \omega)$.

Example 3.6. Take the six-dimensional completely solvable solvmanifold

$$
X:=(-23,0,0,-46,56,0)
$$

endowed with the linear symplectic structure

$$
\omega:=e^{12}+e^{36}+e^{45}
$$

By Hattori's theorem [19, Corollary 4.2], one computes

$$
\begin{aligned}
& H_{d R}^{1}(X ; \mathbb{R})=\underbrace{\mathbb{R}\left\langle e^{2}, e^{3}, e^{6}\right\rangle}_{=H_{\omega}^{(0,1)}(X ; \mathbb{R})}, \\
& H_{d R}^{2}(X ; \mathbb{R})=\underbrace{\mathbb{R}\left\langle e^{12}+e^{36}+e^{45}\right\rangle}_{=H_{\omega}^{(1,0)}(X ; \mathbb{R})} \oplus \underbrace{\mathbb{R}\left\langle e^{12}-e^{36}, e^{12}-e^{45}, e^{13}, e^{26}\right\rangle}_{=H_{\omega}^{(0,2)}(X ; \mathbb{R})}, \\
& H_{d R}^{3}(X ; \mathbb{R})=\mathbb{R}\left\langle e^{123}, e^{126}, e^{136}, e^{245}, e^{345}, e^{456}\right\rangle .
\end{aligned}
$$

Note that, $e^{245}+\mathrm{d} e^{16}$ being primitive,

$$
H_{\omega}^{(0,3)}(X ; \mathbb{R}) \supseteq \mathbb{R}\left\langle e^{123}-e^{345}, e^{126}-e^{456}, e^{245}\right\rangle,
$$

and, being $e^{245}-\mathrm{d} e^{16}=L e^{2}$,

$$
H_{\omega}^{(1,1)}(X ; \mathbb{R})=L H_{\omega}^{(0,3)}(X ; \mathbb{R}) \supseteq \mathbb{R}\left\langle e^{123}+e^{345}, e^{126}+e^{456}, e^{245}\right\rangle,
$$

while, being

$$
e^{136}=\underbrace{\frac{1}{2}\left(e^{136}+e^{145}\right)}_{\in L \mathrm{P} \wedge \wedge^{1} X}+\underbrace{\frac{1}{2}\left(e^{136}-e^{145}\right)}_{\in \mathrm{P} \wedge^{3} X}
$$


and

$$
\mathrm{d} \wedge^{2} \mathfrak{g}^{*}=\mathbb{R}\left\langle e^{146}-e^{234}, e^{156}+e^{235}, e^{236}, e^{246}, e^{256}, e^{346}, e^{356}\right\rangle,
$$

one has

$$
\left\langle e^{136}\right\rangle \notin H_{\omega}^{(0,3)}(X ; \mathbb{R})+H_{\omega}^{(1,1)}(X ; \mathbb{R}),
$$

hence $H_{\omega}^{(0,3)}(X ; \mathbb{R})+H_{\omega}^{(1,1)}(X ; \mathbb{R}) \subsetneq H_{d R}^{3}(X ; \mathbb{R})$.

The next example gives explicit examples of dual currents on a closed symplectic half-flat manifold.

Example 3.7. Let $\mathbb{C}^{3}$ be endowed with the product $*$ defined by

$$
\left(w^{1}, w^{2}, w^{3}\right) *\left(z^{1}, z^{2}, z^{3}\right)=\left(z^{1}+w^{1}, \mathrm{e}^{w^{1}} z^{2}+w^{2}, \mathrm{e}^{w^{1}} z^{3}+w^{3}\right)
$$

for every $\left(w^{1}, w^{2}, w^{3}\right),\left(z^{1}, z^{2}, z^{3}\right) \in \mathbb{C}^{3}$. Then $\left(\mathbb{C}^{3}, *\right)$ is a complex solvable (non-nilpotent) Lie group and, according to $[\mathbf{2 7}]$, it admits lattice $\Gamma \subset \mathbb{C}^{3}$, such that $X=\Gamma \backslash\left(\mathbb{C}^{3}, *\right)$ is a solvmanifold. Setting

$$
\varphi^{1}:=\mathrm{d} z^{1}, \quad \varphi^{2}:=\mathrm{e}^{z^{1}} \mathrm{~d} z^{2}, \quad \varphi^{3}:=\mathrm{e}^{-z^{1}} \mathrm{~d} z^{3},
$$

then $\left\{\varphi^{1}, \varphi^{2}, \varphi^{3}\right\}$ is a global complex co-frame on $X$ satisfying the following complex structure equations:

$$
\mathrm{d} \varphi^{1}=0, \quad \mathrm{~d} \varphi^{2}=\varphi^{12}, \quad \mathrm{~d} \varphi^{3}=-\varphi^{13} .
$$

If we set $\varphi^{j}=: e^{j}+\mathrm{i} e^{3+j}$, for $j \in\{1,2,3\}$, then the last equations yield to

$$
\left\{\begin{array}{l}
\mathrm{d} e^{1}=\mathrm{d} e^{4}=0 \\
\mathrm{~d} e^{2}=e^{12}-e^{45} \\
\mathrm{~d} e^{3}=-e^{13}+e^{46} \\
\mathrm{~d} e^{5}=e^{15}-e^{24} \\
\mathrm{~d} e^{6}=-e^{16}+e^{34}
\end{array}\right.
$$

Then (see [12]),

$$
\omega:=e^{14}+e^{35}+e^{62}
$$

and

$$
\begin{gathered}
J e^{1}:=-e^{4}, \quad J e^{3}:=-e^{5}, \quad J e^{6}:=-e^{2}, \\
J e^{4}:=e^{1}, \quad J e^{5}:=e^{3}, \quad J e^{2}:=e^{6},
\end{gathered}
$$

and

$$
\psi:=\left(e^{1}+\mathrm{i} e^{4}\right) \wedge\left(e^{3}+\mathrm{i} e^{5}\right) \wedge\left(e^{6}+\mathrm{i} e^{2}\right)
$$

give rise to a symplectic half-flat structure on $X$, where

$$
\Re \mathfrak{e} \psi=e^{136}+e^{125}+e^{234}-e^{456} .
$$

Note that the Hard Lefschetz Condition holds on $(X, \omega)$, see [12, Theorem 5.1]. 
Then, setting $z^{j}=: x^{j}+\mathrm{i} y^{j}$, for $j \in\{1,2,3\}$, and denoting by $\pi: \mathbb{C}^{3} \rightarrow X$ the canonical projection, we easily check that

$$
\begin{aligned}
& Y_{1}:=\pi\left(\left\{\left(x^{1}, x^{2}, x^{3}, y^{1}, y^{2}, y^{3}\right) \in \mathbb{C}^{3}: x^{2}=y^{1}=y^{2}=0\right\}\right), \\
& Y_{2}:=\pi\left(\left\{\left(x^{1}, x^{2}, x^{3}, y^{1}, y^{2}, y^{3}\right) \in \mathbb{C}^{3}: x^{3}=y^{1}=y^{3}=0\right\}\right)
\end{aligned}
$$

are compact oriented special Lagrangian submanifolds of $(X, \omega, \psi)$, namely, for $j \in\{1,2\}$, it holds $\Re \mathfrak{e} \psi\left\lfloor_{Y_{j}}=\mathrm{Vol}_{Y_{j}}\right.$, and, consequently, the associated dual currents $\rho_{Y_{j}}$ are primitive.

\section{References}

[1] D. Angella and F.A. Rossi, Cohomology of D-complex manifolds, Differ. Geom. Appl. 30(5) (2012), 530-547.

[2] D. Angella and A. Tomassini, On cohomological decomposition of almost-complex manifolds and deformations, J. Symplec. Geom. 9(3) (2011), 403-428.

[3] D. Angella and A. Tomassini, On the cohomology of almost-complex manifolds, Int. J. Math. 23(2) (2012), 1250019, 25 pp.

[4] D. Angella and A. Tomassini, On the $\partial \bar{\partial}-$ Lemma and Bott-Chern cohomology, Invent. Math. 192(1) (2013), 71-81.

[5] F.A. Belgun, On the metric structure of non-Kähler complex surfaces, Math. Ann. $\mathbf{3 1 7}(1)$ (2000), 1-40.

[6] Ch. Benson and C. S. Gordon, Kähler and symplectic structures on nilmanifolds, Topology 27(4) (1988), 513-518.

[7] C. Bock, On low-dimensional solvmanifolds, arXiv:0903.2926v4 [math.DG].

[8] J.-L. Brylinski, A differential complex for Poisson manifolds, J. Differ. Geom. 28(1) (1988), 93-114.

[9] G. Cavalcanti, New aspects of the dd $d^{c}$-lemma, Oxford University D. Phil thesis, arXiv:math/0501406v1 [math.DG].

[10] S. Chiossi and S. Salamon, The Intrinsic Torsion of SU(3) and $G_{2}$ Structures, Differential Geometry, Valencia, 2001, 115-133, World Scientific Publ., River Edge, NJ, 2002.

[11] D. Conti and A. Tomassini, Special symplectic six-manifolds, Q. J. Math. 58(3) (2007), 297-311.

[12] P. de Bartolomeis and A. Tomassini, On solvable generalized Calabi-Yau manifolds, Ann. Inst. Fourier 56(5) (2006), 1281-1296.

[13] P. Deligne, Ph. Griffiths, J. Morgan and D.P. Sullivan, Real homotopy theory of Kähler manifolds, Invent. Math. 29(3) (1975), 245-274.

[14] G. de Rham, Differentiable manifolds, Forms, currents, harmonic forms, Translated from the French by F.R. Smith, With an introduction by S.S. Chern, Grundlehren der Mathematischen Wissenschaften [Fundamental Principles of Mathematical Sciences], Vol. 266, Springer-Verlag, Berlin, 1984. 
[15] T. Drăghici, T.-J. Li and W. Zhang, Symplectic forms and cohomology decomposition of almost complex four-manifolds, Int. Math. Res. Not. IMRN 2010(1) (2010), 1-17.

[16] A. Fino and G. Grantcharov, On some properties of the manifolds with skewsymmetric torsion, Adv. Math. 189(2) (2004), 439-450.

[17] A. Fino and A. Tomassini, On some cohomological properties of almost complex manifolds, J. Geom. Anal. 20(1) (2010), 107-131.

[18] V. Guillemin, Symplectic Hodge theory and the d $\delta$-Lemma, preprint, Massachusetts Institute of Technology, 2001.

[19] A. Hattori, Spectral sequence in the de Rham cohomology of fibre bundles, J. Fac. Sci. Univ. Tokyo Sect. I 8(1960) (1960), 289-331.

[20] D. Huybrechts, Complex Geometry. An Introduction, Universitext, Springer-Verlag, Berlin, 2005.

[21] T.-J. Li, W. Zhang, Comparing tamed and compatible symplectic cones and cohomological properties of almost complex manifolds, Commun. Anal. Geom. 17(4) (2009), 651-684.

[22] Y. Lin, Symplectic Harmonic theory and the Federer-Fleming deformation theorem, arXiv: 1112.2442v3 [math.SG].

[23] A.I. Mal'tsev, On a class of homogeneous spaces, Izvestiya Akad. Nauk. SSSR. Ser. Mat. 13(1) (1949), 9-32,

English translation in Amer. Math. Soc. Translation: Series 1 1951(39) (1951), 193-206.

[24] O. Mathieu, Harmonic cohomology classes of symplectic manifolds, Comment. Math. Helv. 70(1) (1995), 1-9.

[25] S.A. Merkulov, Formality of canonical symplectic complexes and Frobenius manifolds, Int. Math. Res. Not. 1998(14) (1998), 727-733.

[26] J. Milnor, Curvature of left-invariant metrics on Lie groups, Adv. in Math. 21(3) (1976), 293-329.

[27] I. Nakamura, Complex parallelisable manifolds and their small deformations, J. Differ. Geom. 10(1) (1975), 85-112.

[28] K. Nomizu, On the cohomology of compact homogeneous spaces of nilpotent Lie groups, Ann. Math. (2) 59(3) (1954), 531-538.

[29] L.-S. Tseng and S.-T. Yau, Cohomology and Hodge theory on symplectic manifolds: I, J. Differ. Geom. 91(3) (2012), 383-416.

[30] L.-S. Tseng and S.-T. Yau, Cohomology and Hodge theory on symplectic manifolds: II, J. Differ. Geom. 91(3) (2012), 417-443.

[31] L.-S. Tseng and S.-T. Yau, Generalized cohomologies and supersymmetry, arXiv: 1111.6968v1 [hep-th].

[32] D. Yan, Hodge structure on symplectic manifolds, Adv. Math. 120(1) (1996), 143-154.

(Daniele Angella) Dipartimento di Matematica

UNIVERSITÀ DI PISA

Largo Bruno Pontecorvo 5

56127 PISA

ITALY

E-mail address: angella@mail.dm.unipi.it 
Current address: Istituto Nazionale di Alta Matematica

at Dipartimento di Matematica e Informatica

Universitá Di PARMA

Parco Area delle Scienze 53/A

43124 PARMA

ITALY.

E-mail address: daniele.angella@math.unipr.it

(Adriano Tomassini) Dipartimento di Matematica e Informatica

Università di PARMA

Parco Area delle Scienze 53/A

43124 PARMA

ITALY

E-mail address: adriano.tomassini@unipr.it

Received 04/18/2012, accepted 02/04/2013

The authors would like to thank Yi Lin for pointing them the reference $[\mathbf{2 2}]$, and for useful suggestions. We are also pleased to thank the anonymous referee for many fruitful suggestions and remarks that improved the presentation of the results.

This work was supported by the Project PRIN "Varietá reali e complesse: geometria, topologia e analisi armonica", by the Project FIRB "Geometria Differenziale e Teoria Geometrica delle Funzioni" and by GNSAGA of INdAM 